\title{
Numerical Simulation and Theoretical Modeling of Longitudinal Compressive Failure in Fiber Reinforced Composite Materials
}

\author{
Takeaki Nadabe \\ Department of Advanced Energy \\ The University of Tokyo \\ Chiba, Japan \\ e-mail: nadabe@smart.k.u-tokyo.ac.jp
}

\author{
Nobuo Takeda \\ Department of Advanced Energy \\ The University of Tokyo \\ Chiba, Japan \\ e-mail: takeda@smart.k.u-tokyo.ac.jp
}

\begin{abstract}
This study investigates the numerical simulation and theoretical modeling of longitudinal compressive failure in fiber reinforced composite materials. Firstly the numerical simulation of longitudinal compressive failure is conducted. The simulated results show that at one moment of the loading, the localized deformation catastrophically appears in the material, and in this initiation of the localized deformation, the reduction of tangent shear stiffness plays an important role. Secondly the theoretical modeling of longitudinal compressive failure is implemented. A set of mathematical equations is obtained for the deformation of composite materials, and the mathematical solution of the equations is considered. There exists a state where arbitrariness appears in the solution of equations expressing deformation of composite materials, and it is indicated that the onset of arbitrariness in solution of equations expressing deformation of composite materials is closely related with the initiation of longitudinal compressive failure, and also related with the initiation of narrow localized band in the materials. Finally the numerical simulation is conducted for compressive failure in quasi-isotropic laminate. The localized deformation also appears in the laminate, and the simulated deformation of the material agrees with the microscope picture of the experimental result.
\end{abstract}

Keywords-numerical simulation; theoretical modeling; fracture mechanism; strength analysis; composite materials

\section{INTRODUCTION}

Composite materials commonly have complex internal structures including fibers, matrix, interfaces and interlaminar regions, and when precise evaluation of fracture strength of the material is conducted, the internal fracture process in the materials is necessary to be taken into account in the numerical analysis [1]. In recent years, composite materials are being increasingly used in several industrial fields, and the precise evaluation of mechanical response of the material under various loading condition and environmental condition increases the necessity in design and improvement of industrial products [2]. Compressive failure is one of the typical failure modes in fiber reinforced composite materials [3-4], and fracture strength in compressive failure often becomes one of the limiting factors at the design phase of structural elements [5]. Not only uniaxial compressive strength but also compressive strength at around open holes and post-impact compressive strength in the materials are related to the fundamental compressive strength of the materials, and improvement of compressive strength would be related with the increase of the light weight potential of the materials. This study investigates the numerical simulation and theoretical modeling of longitudinal compressive failure in fiber reinforced composite materials.

\section{NUMERICAL SIMULATION OF LONGITUDINAL COMPRESSIVE FAILURE}

\section{A. Numerical Model}

Firstly the numerical simulation of longitudinal compressive failure is conducted. Finite element method is used to simulate the longitudinal compressive failure. Fig. 1 shows the numerical model of this analysis. The white and gray elements in Fig. 1 represent fibers and matrix, respectively. The thickness of the ply in y-direction is 600 $\mu \mathrm{m}$. The length in $\mathrm{x}$-direction is $1000 \mu \mathrm{m}$, and the thickness in $\mathrm{z}$-direction is $100 \mathrm{~mm}$. The diameter of each fiber is set to $3.5 \mu \mathrm{m}$, and the interval of fibers is $11.9 \mu \mathrm{m}$. The fiber volume fraction of the materials is set to $29.8 \%$. Each fiber and matrix is modeled by two-dimensional plate elements. The elements have eight nodes and four integration points in order to avoid the shear locking and zero-energy mode deformation particularly in plastic deformation. The one fiber placed at the center has the initial misalignment as shown in Fig. 1. The initial misalignment of the fiber is introduced using the sine function. The x coordinate of each node is placed regularly at the interval of $5.0 \mu \mathrm{m}$, and the y coordinate of each node is calculated using the sine function. Only the central part of this fiber has the misalignment and the other part of the fiber is modeled as the straight line. The other fibers are also modeled as the straight lines and the fiber axial direction is parallel to the $\mathrm{x}$-direction.

Due to the atomic structure in the inside of the fibers, the fibers commonly have the different material property in between fiber axial and transverse directions. Here, the fibers are modeled by the transversely isotropic elastic material. Table I shows the material property of the fibers. Carbon fiber AS4 (Hexcel Corp.) is assumed [6]. Matrix is modeled by isotropic elastic-plastic material. Commonly the compressive failure of composite materials is affected by the nonlinear stress-strain relation of matrix, thus in this 
analysis the nonlinear stress-strain curve of matrix shown in Fig. 2 (hardening M) is applied, and the nonlinear finite element analysis is conducted. Table II shows the material property of matrix. Epoxy resin 3501-6 (Hercules Chemical Company, Inc.) is assumed [6]. The quasi-static and room temperature environment are assumed in the analysis.

Since the geometrical nonlinearity commonly affects the buckling phenomena of the materials, the geometrical nonlinear effect is incorporated in the analysis. The incremental analysis in the finite element analysis is conducted by the arc-length method. In the initial increment, the average applied strain to the material in $\mathrm{x}$ direction is set to $0.002 \%$. The analysis is conducted until the average applied strain $2.0 \%$. The domain decomposition method is applied to conduct the parallel

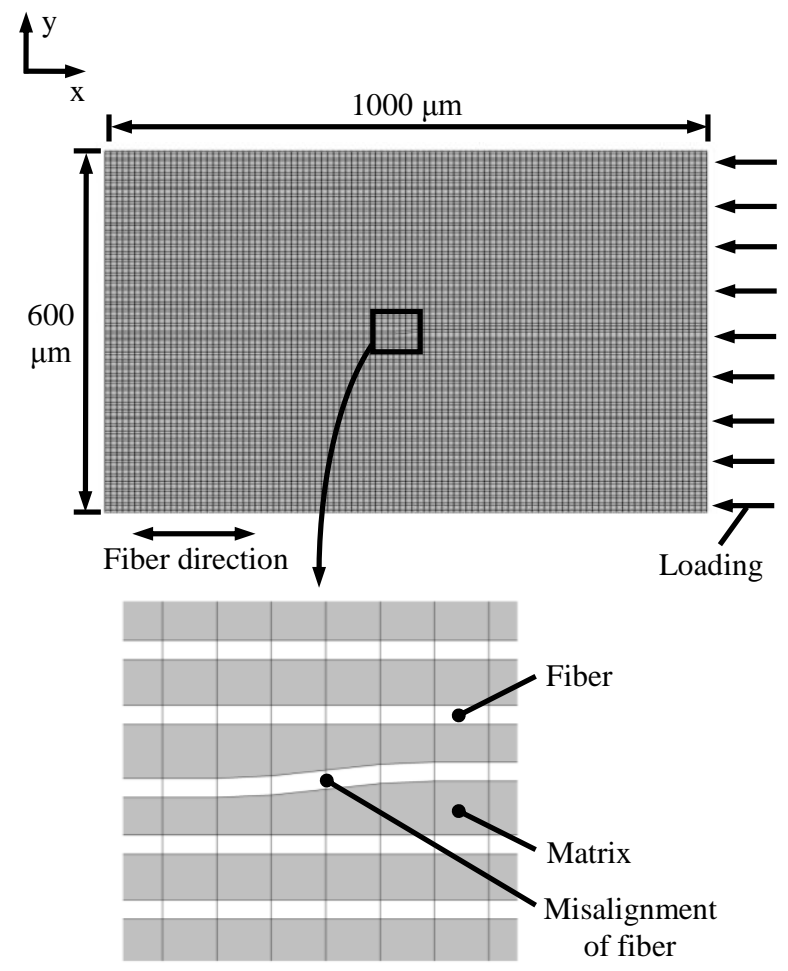

Figure 1. Numerical model for longitudinal compressive failure.

TABLE I. MATERIAL PROPERTY OF FibER. CARBON Fiber AS4 (HEXCEL CORP.) Is ASSUMED [6].

\begin{tabular}{|l|c|c|}
\hline Elastic modulus in fiber axial direction & 225 & $\mathrm{GPa}$ \\
\hline Elastic modulus in transverse direction & 15 & $\mathrm{GPa}$ \\
\hline In-plane Poisson's ratio & 0.20 & \\
\hline In-plane shear modulus & 15 & $\mathrm{GPa}$ \\
\hline Transverse shear modulus & 7.0 & $\mathrm{GPa}$ \\
\hline
\end{tabular}

TABLE II. MATERIAL PROPERTY OF MATRIX. EPOXY RESIN 3501-6 (HERCULES CHEMICAL COMPANY, INC.) Is ASSUMEd [6].

\begin{tabular}{|l|c|c|}
\hline Elastic modulus & 4.2 & $\mathrm{GPa}$ \\
\hline Poisson's ratio & 0.34 & \\
\hline Yield stress & 90 & $\mathrm{MPa}$ \\
\hline
\end{tabular}

computing in the numerical calculation. The authors produced fortran program for this analysis, and the analysis is conducted using this program.

\section{B. Simulated Results and Discussions}

Figs. 3 and 4 show the simulated results of deformation and stress distribution of the material, respectively. Simulated results show that in the initial state of the loading, the stress concentration occurs in the material around the initial misalignment of fiber, and when the app-

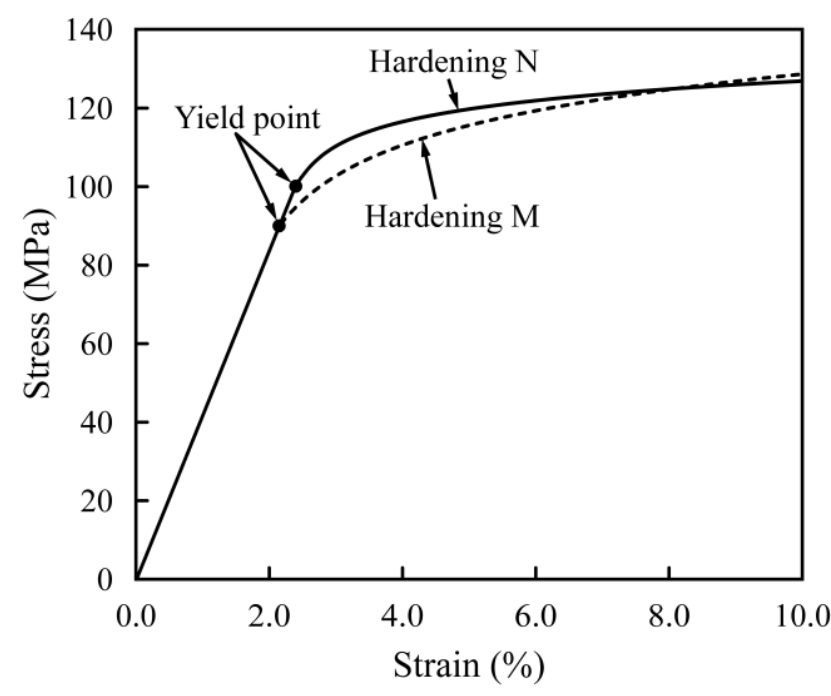

Figure 2. Stress-strain curve of matrix.

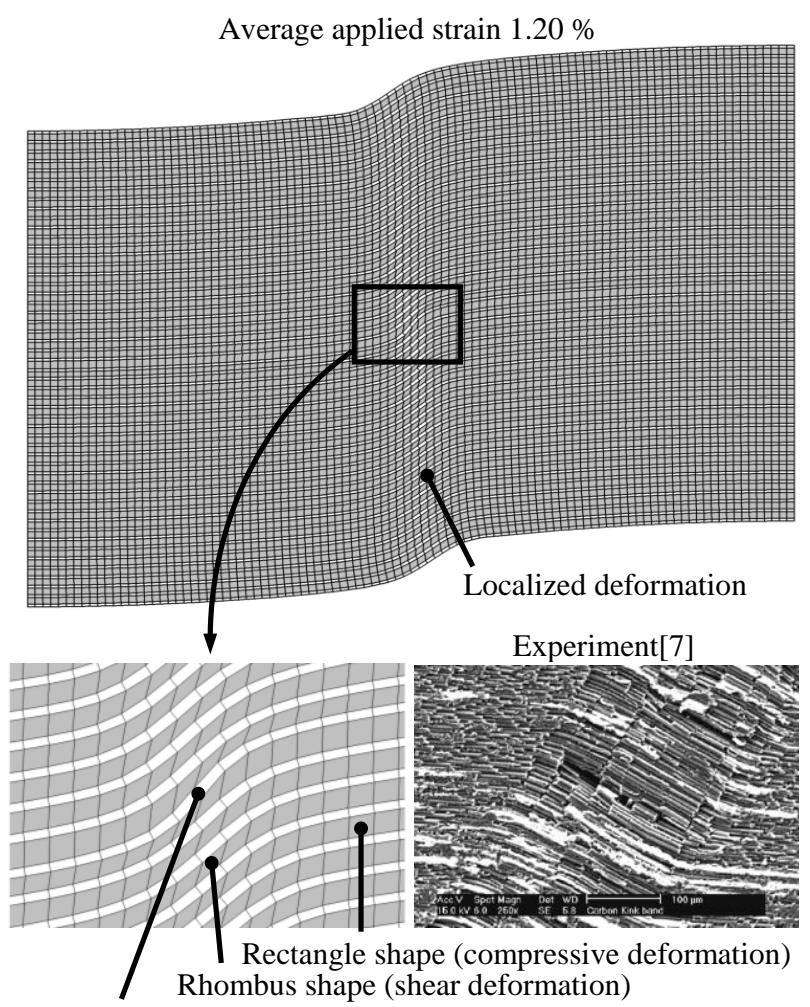

Microbuckling of fiber

Figure 3. Simulated results of deformation in longitudinal compressive failure. 

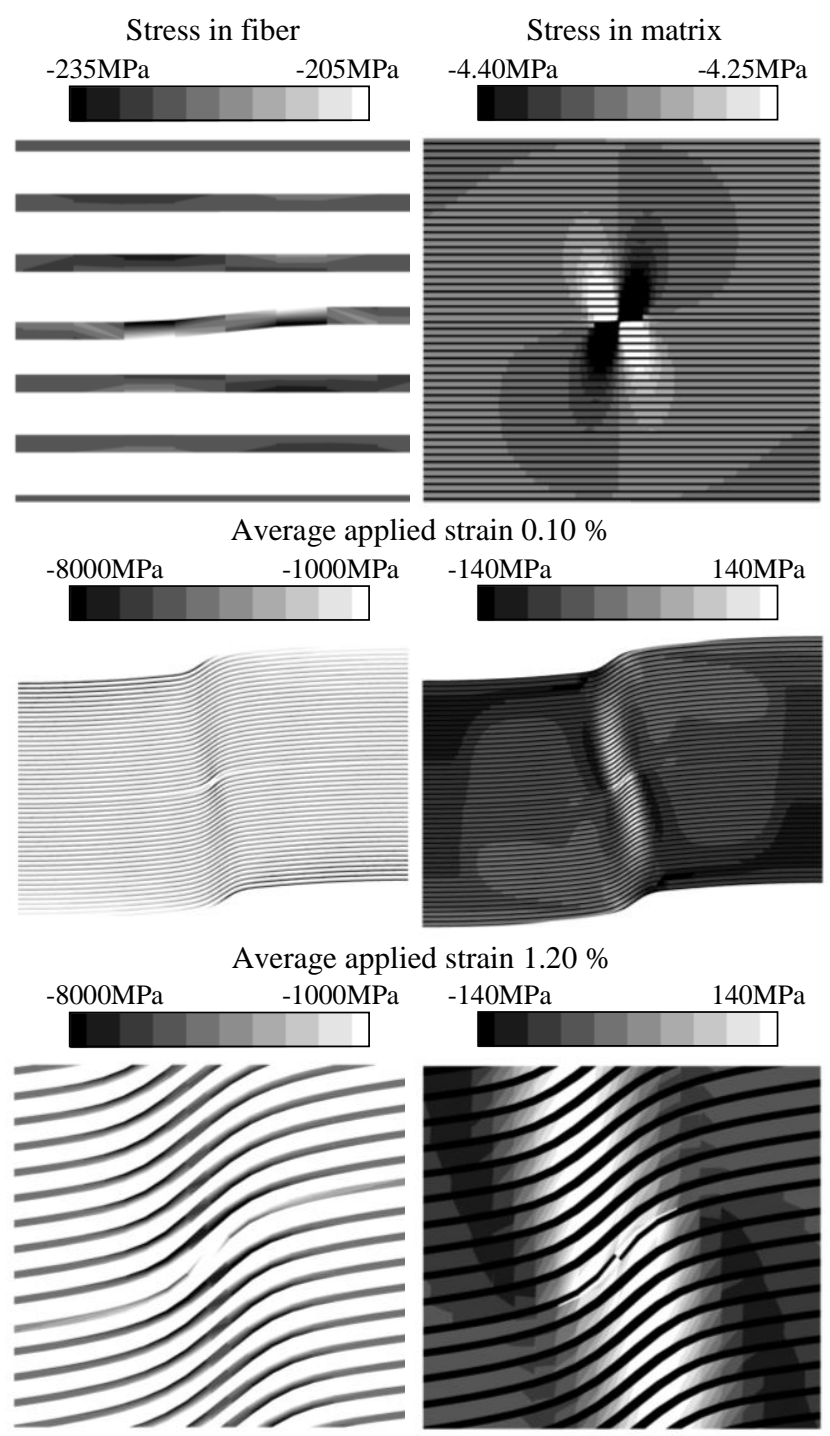

Average applied strain $1.20 \%$ (Magnified picture)

Figure 4. Simulated results of stress distribution in longitudinal compressive failure.

lied load is increased, local areas of matrix around the stress concentration start to yield, and deformation is locally increased. At one moment of the loading, a large deformation occurs within a narrow band, and a band of localized deformation develops rapidly. This band of localized deformation passes across the misalignment part of center fiber. As shown in the figures, fibers cause bending deformation, and fiber direction is largely rotated. Matrix causes shear deformation, and the shape of the elements is close to rhombus shape which is rectangle shape in initial state. After the yielding of matrix, the elastic-plastic tangent shear stiffness of matrix significantly reduces, and the shear strain rapidly increases. Then the shear deformation of this part of matrix increases, and due to the shear deformation of the part, the band of localized deformation is formed. The reduction of shear stiffness of matrix is the essential factor in the initiation of the localized deformation of the material.

\section{THEORETICAL MODELING OF LONGITUDINAL COMPRESSIVE FAILURE}

\section{A. Equations Expressing Deformation of Composite Materials}

Here, the equations expressing deformation of composite materials are compiled. The equations consist of motion equation and constitutive equation. The motion equation is represented as the following,

$$
\rho_{0} \frac{\partial^{2} u_{i}}{\partial t^{2}}=\frac{\partial P_{i j}}{\partial X_{j}}+\rho_{0} f_{i}
$$

where $\rho_{0}$ is density, $t$ is time, $u_{i}$ is displacement, $X_{j}$ is coordinate at reference configuration, $P_{i j}$ is the first Piola-Kirchhoff stress and $f_{i}$ is external force. The nonlinear stress-strain relation of composite materials is represented by the nonlinear deformation theory shown by Tohgo et al. [8].

$$
\begin{gathered}
d \boldsymbol{\sigma}=\boldsymbol{C}_{\text {comp }} d \boldsymbol{\varepsilon} \\
\boldsymbol{C}_{\text {comp }}=\boldsymbol{C}_{m}\left\{\left(1-V_{f}\right)\left(\boldsymbol{C}_{f}-\boldsymbol{C}_{m}\right) \boldsymbol{S}+\boldsymbol{C}_{m}\right\}^{-1} \boldsymbol{K} \\
\left.\boldsymbol{K}=\left(1-V_{f}\right)\left(\boldsymbol{C}_{f}-\boldsymbol{C}_{m}\right) \boldsymbol{S}+\boldsymbol{C}_{m}\right\}+V_{f} \boldsymbol{C}_{f}
\end{gathered}
$$

where $d \boldsymbol{\sigma}$ is stress rate, $d \boldsymbol{\varepsilon}$ is strain rate, $\boldsymbol{C}_{\text {comp }}, \boldsymbol{C}_{f}$ and $\boldsymbol{C}_{m}$ are constitutive tensors of composites, fibers and matrix, respectively, $V_{f}$ is fiber volume fraction and $\boldsymbol{S}$ is Eshelby tensor. In order to apply the stress-strain relation in (2) in numerical analysis, evaluation of equivalent stress of matrix is necessary. The following relation is applied to evaluate the equivalent stress of matrix from the applied stress in composite materials.

$$
d \boldsymbol{\sigma}_{m}=\boldsymbol{C}_{m}(\boldsymbol{S}-\boldsymbol{I}) \boldsymbol{K}^{-\boldsymbol{I}}\left\{\boldsymbol{C}_{m}+\left(\boldsymbol{C}_{f}-\boldsymbol{C}_{m}\right) \boldsymbol{S}\right\}(\boldsymbol{S}-\boldsymbol{I})^{-1} \boldsymbol{C}_{m}{ }^{-1} d \boldsymbol{\sigma}
$$

where $d \boldsymbol{\sigma}_{m}$ is stress rate of matrix and $\boldsymbol{I}$ is unit tensor. Next, the effect of geometrical nonlinearity during the material deformation is considered. Here the constitutive tensor in spacial description is defined in the relation between the second Piola-Kirchhoff stress and the right Cauchy-Green deformation tensor.

$$
C_{a b c d}^{s p a}=\frac{\partial S_{a b}}{\partial C_{c d}^{C G}}
$$

where $C_{a b c d}^{s p a}$ is constitutive tensor in spacial description, $S_{a b}$ is the second Piola-Kirchhoff stress and $C_{c d}^{C G}$ is the right Cauchy-Green deformation tensor. The constitutive tensor in material description is represented by the constitutive tensor in spacial description as follows,

$$
\begin{aligned}
C_{i j k l}^{m a t} & =2 J^{-1} F_{i a} F_{j b} F_{k c} F_{l d} C_{a b c d}^{s p a} \\
& =2 \frac{1}{J} \frac{\partial x_{i}}{\partial X_{a}} \frac{\partial x_{j}}{\partial X_{b}} \frac{\partial x_{k}}{\partial X_{c}} \frac{\partial x_{l}}{\partial X_{d}} C_{a b c d}^{s p a}
\end{aligned}
$$

where $C_{i j k l}^{m a t}$ is constitutive tensor in material description, $F_{i a}$ is deformation gradient, $J=\operatorname{det} F_{i j}$ is Jacobian and 
$x_{i}$ is coordinate at present configuration. Cauchy stress is represented by the second Piola-Kirchhoff stress, deformation gradient and Jacobian as follows,

$$
\begin{gathered}
\sigma_{i j}=J^{-1} F_{i k} S_{k l} F_{j l} \\
\dot{\sigma}_{i j}=J^{-1} \dot{F}_{i k} S_{k l} F_{j l}+J^{-1} F_{i k} \dot{S}_{k l} F_{j l} \\
+J^{-1} F_{i k} S_{k l} \dot{F}_{j l}-\dot{J} J^{-1} F_{i k} S_{k l} F_{j l}
\end{gathered}
$$

where $\sigma_{i j}$ is Cauchy stress and $\dot{\sigma}_{i j}$ is the material time derivative of Cauchy stress. Here, the time derivative of deformation gradient and Jacobian is

$$
\dot{F}_{i j}=L_{i k} F_{k j}, \dot{J}=L_{i i}
$$

where $L_{i k}$ is velocity gradient. Then

$$
\begin{aligned}
\dot{\sigma}_{i j}= & J^{-1} L_{i m} F_{m k} S_{k l} F_{j l}+J^{-1} F_{i k} \dot{S}_{k l} F_{j l} \\
& +J^{-1} F_{i k} S_{k l} F_{m l} L_{j m}-J^{-1} L_{m m} F_{i k} S_{k l} F_{j l} \\
= & J^{-1} F_{i k} \dot{S}_{k l} F_{j l}+L_{i k} \sigma_{k j}+\sigma_{i k} L_{j k}-\sigma_{i j} L_{l l}
\end{aligned}
$$

where

$$
\begin{gathered}
\dot{S}_{k l}=C_{k l m n}^{s p a} \cdot \dot{C}_{m n}^{C G}=C_{k l m n}^{s p a} \dot{F}_{o m} F_{o n}+C_{k l m n}^{s p a} F_{o m} \dot{F}_{o n} \\
=C_{k l m n}^{s p a} L_{o p} F_{p m} F_{o n}+C_{k l m n}^{s p a} F_{o m} L_{o p} F_{p n} \\
J^{-1} F_{i k} \dot{S}_{k l} F_{j l}= \\
J^{-1} F_{i k} F_{j l} F_{p m} F_{o n} C_{k l m n}^{s p a} L_{o p} \\
+J^{-1} F_{i k} F_{j l} F_{o m} F_{p n} C_{k l m n}^{s p a} L_{o p} \\
=\frac{1}{2} C_{i j p o}^{m a t} L_{o p}+\frac{1}{2} C_{i j o p}^{m a t} L_{o p} \\
=C_{i j k l}^{m a t} \cdot \frac{1}{2}\left(L_{l k}+L_{k l}\right)=C_{i j k l}^{m a t} D_{k l}
\end{gathered}
$$

Therefore

$$
\dot{\sigma}_{i j}=C_{i j k l}^{m a t} D_{k l}+L_{i k} \sigma_{k j}+\sigma_{i k} L_{j k}-\sigma_{i j} L_{l l}
$$

This coinsides with the formulation of Truesdell rate of Cauchy stress. Therefore, here the formulation of finite deformation is based on Truesdell rate of Cauchy stress. Then the rate of the first Piola-Kirchhoff stress is represented as follows,

$$
\begin{aligned}
\dot{P}_{i j} & =J \frac{\partial X_{j}}{\partial x_{k}}\left(\dot{\sigma}_{i k}+\sigma_{i k} L_{l l}-\sigma_{i l} L_{k l}\right) \\
& =J \frac{\partial X_{j}}{\partial x_{m}}\left(C_{i m k l}^{m a t} D_{k l}+\sigma_{l m} L_{i l}\right) \\
& =J \frac{\partial X_{j}}{\partial x_{m}}\left(C_{i m k l}^{m a t}+\sigma_{l m} \delta_{i k}\right) \frac{\partial \dot{u}_{k}}{\partial x_{l}}
\end{aligned}
$$

where $\delta_{i k}$ is Kronecker delta. From (1) and (13), a set of equations expressing deformation of composite materials is obtained.

$$
\begin{gathered}
\rho_{0} \frac{\partial^{2} u_{i}}{\partial t^{2}}=\frac{\partial P_{i j}}{\partial X_{j}}+\rho_{0} f_{i} \\
\dot{P}_{i j}=J \frac{\partial X_{j}}{\partial x_{m}}\left(C_{i m k l}^{m a t}+\sigma_{l m} \delta_{i k}\right) \frac{\partial \dot{u}_{k}}{\partial x_{l}}
\end{gathered}
$$

\section{B. Arbitrariness Appearing in Solution of Equations} Expressing Deformation of Composite Materials

Equations (14) and (15) are unified to one differential equation.

$$
\rho_{0} \frac{\partial^{2} \dot{u}_{i}}{\partial t^{2}}-\rho_{0} \dot{f}_{i}=\frac{\partial}{\partial X_{j}}\left(A_{i j k l} \frac{\partial \dot{u}_{k}}{\partial x_{l}}\right)
$$

where tensor $A_{i j k l}$ is

$$
A_{i j k l}=J \frac{\partial X_{j}}{\partial x_{m}}\left(C_{i m k l}^{m a t}+\sigma_{l m} \delta_{i k}\right)
$$

Equation (16) plays a role of governing equation in the deformation of composite materials. When the reference configuration is taken at the moment of the present time, and in the place where the external force doesn't act, (16) becomes as follows,

$$
\rho \frac{\partial^{2} \dot{u}_{i}}{\partial t^{2}}=\frac{\partial}{\partial x_{j}}\left(A_{i j k l} \frac{\partial \dot{u}_{k}}{\partial x_{l}}\right)
$$

where $\rho$ is density at the present time. Here, we conduct the transformation of coordinate system for this equation. Firstly each variable is transformed as the following in the transformation of coordinate system.

$$
\begin{gathered}
d x_{i}=\frac{\partial x_{i}}{\partial x_{a}^{\prime}} d x_{a}^{\prime}, \dot{u}_{i}=\frac{\partial x_{i}}{\partial x_{a}^{\prime}} \dot{u}_{a}^{\prime}, \frac{\partial}{\partial x_{l}}=\frac{\partial x_{d}^{\prime}}{\partial x_{l}} \frac{\partial}{\partial x_{d}^{\prime}} \\
L_{k l}=\frac{\partial x_{k}}{\partial x_{c}^{\prime}} \frac{\partial x_{d}^{\prime}}{\partial x_{l}} \frac{\partial u_{c}^{\prime}}{\partial x_{d}^{\prime}}=\frac{\partial x_{k}}{\partial x_{c}^{\prime}} \frac{\partial x_{d}^{\prime}}{\partial x_{l}} L_{c d}^{\prime}, \sigma_{i j}=\frac{\partial x_{i}}{\partial x_{a}^{\prime}} \frac{\partial x_{j}}{\partial x_{b}^{\prime}} \sigma_{a b}^{\prime} \\
A_{i j k l}=\frac{\partial x_{i}}{\partial x_{a}^{\prime}} \frac{\partial x_{j}}{\partial x_{b}^{\prime}} \frac{\partial x_{c}^{\prime}}{\partial x_{k}} \frac{\partial x_{l}}{\partial x_{d}^{\prime}} A_{a b c d}^{\prime}
\end{gathered}
$$

where $x_{a}^{\prime}$ is the coordinate system after the transformation. Then (18) is transformed as follows,

$$
\rho \frac{\partial^{2} \dot{u}_{a}^{\prime}}{\partial t^{2}}=\frac{\partial}{\partial x_{b}^{\prime}}\left(A_{a b c d}^{\prime} \frac{\partial \dot{u}_{c}^{\prime}}{\partial x_{d}^{\prime}}\right)
$$

Commonly the governing equations for natural phenomena do not change their form in the coordinate transformation. Next, when the deformation is locally isotropic in $2^{\prime}$ and $3^{\prime}$ directions, $\partial / \partial x_{2}^{\prime}$ and $\partial / \partial x_{3}^{\prime}$ are equal to zero, and when the deformation is quasi-static, 
$\partial / \partial t$ becomes equal to zero, which corresponds with the case when inertia term is infinitesimal, then (20) becomes as follows,

$$
\frac{\partial}{\partial x_{1}^{\prime}}\left(A_{a 1 c 1}^{\prime} \frac{\partial \dot{u}_{c}^{\prime}}{\partial x_{1}^{\prime}}\right)=0
$$

Here, the eigenvalue problem of the tensor $A_{a 1 c 1}^{\prime}$ is considered. Using the eigenvalue $\lambda^{\prime}$ and the eigenvector $v_{c}^{\prime}$ of the tensor $A_{a 1 c 1}^{\prime}$, the eigenvalue problem is represented as

$$
A_{a 1 c 1}^{\prime} v_{c}^{\prime}=\lambda^{\prime} v_{c}^{\prime}
$$

When the tensor $A_{a 1 c 1}^{\prime}$ has zero eigenvalues, (22) becomes as follows,

$$
A_{a 1 c 1}^{\prime} v_{c}^{\prime}=0
$$

Multiplying the arbitrary function $\phi^{\prime}\left(x_{1}^{\prime}\right)$,

$$
A_{a 1 c 1}^{\prime} v_{c}^{\prime} \phi^{\prime}\left(x_{1}^{\prime}\right)=0
$$

Then taking the partial differenciation of $x_{1}^{\prime}$,

$$
A_{a 1 c 1}^{\prime} v_{c}^{\prime} \frac{\partial}{\partial x_{1}^{\prime}} \phi^{\prime}\left(x_{1}^{\prime}\right)=0
$$

This equation means that $\dot{u}_{c}^{\prime}=v_{c}^{\prime} \phi^{\prime}\left(x_{1}^{\prime}\right)$ is one of the solution of (21). Since $\dot{u}_{c}^{\prime}=v_{c}^{\prime} \phi^{\prime}\left(x_{1}^{\prime}\right)$ is the solution of (21) for arbitrary function $\phi^{\prime}\left(x_{1}^{\prime}\right),(21)$ have multiple solutions, or the arbitrariness appears in the solution of (21). This case causes when the tensor $A_{a 1 c 1}^{\prime}$ has zero eigenvalues. When the tensor $A_{a 1 c 1}^{\prime}$ has zero eigenvalues, the determinant of $A_{a 1 c 1}^{\prime}$ becomes zero,

$$
\operatorname{det}\left(A_{a 1 c 1}^{\prime}\right)=0
$$

From (19), the tensor $A_{a 1 c 1}^{\prime}$ is represented by the original coordinate system of tensor $A_{i j k l}$.

$$
A_{a 1 c 1}^{\prime}=A_{i j k l} \frac{\partial x_{a}^{\prime}}{\partial x_{i}} \frac{\partial x_{1}^{\prime}}{\partial x_{j}} \frac{\partial x_{k}}{\partial x_{c}^{\prime}} \frac{\partial x_{1}^{\prime}}{\partial x_{l}}
$$

Here, we introduce two tensors $n_{j}$ and $J_{a i}$ which express the coordinate transformation.

$$
n_{j}=\frac{\partial x_{1}^{\prime}}{\partial x_{j}}, J_{a i}=\frac{\partial x_{a}^{\prime}}{\partial x_{i}}
$$

Then (26) becomes as follows,

$$
\begin{aligned}
\operatorname{det}\left(A_{a 1 c 1}^{\prime}\right) & =\operatorname{det}\left(A_{i j k l} n_{j} n_{l} J_{a i} J_{c k}{ }^{-1}\right) \\
& =\operatorname{det}\left(A_{i j k l} n_{j} n_{l}\right) \cdot \operatorname{det}\left(J_{a i}\right) \cdot \operatorname{det}\left(J_{c k}{ }^{-1}\right)=0
\end{aligned}
$$

Since $\operatorname{det}\left(J_{a i}\right) \neq 0$,

$$
\operatorname{det}\left(A_{i j k l} n_{j} n_{l}\right)=0
$$

As the conclusion of this analysis, when (30) is satisfied, the arbitrariness appears in the solution of (21) which is a specific case of the governing equations for the deformation of composite materials. Equation (30) is considered as the initiation condition of arbitrariness in the solution of the equations for the deformation of composite materials. This is interesting because in structural mechanics it is well recognized that the buckling of the structures is represented by a condition where the determinant of the stiffness matrix of the structures is equal to zero.

$$
\operatorname{det}[K]=0
$$

where $[K]$ is the stiffness matrix. There is a significant similarity in between (30) and (31). In the case of (31), at the time when the equation has equality, the structural instability or the buckling phenomena appear in the structures, and the material and geometrical nonlinearity of the stiffness matrix play important roles in these instability or the buckling. In the case of (30), when the equation has equality, the material instability or the microbuckling phenomena appear in the materials, and the material nonlinearity including the effect of matrix nonlinear stress-strain relation and geometrical nonlinearity including the effect of fiber misalignment play important roles in these instability or the microbuckling. In addition, from (17), (30) also becomes as follows,

$$
\operatorname{det}\left(C_{i j k l}^{m a t} n_{j} n_{l}+\sigma_{j l} \delta_{i k} n_{j} n_{l}\right)=0
$$

The first term of this equation depends on the constitutive tensor of the material, including the elastic and plastic property of the material. It is also related with the material nonlinear effect. The second term of the equation depends on the multi-axial stresses. It is related with the geometrical nonlinear effect. The equation indicates that the appearance of arbitrariness is related with the material property and the multi-axial stresses. The angle of microbuckling is able to affect through the variable $n_{j}$, but the width of the band of the microbuckling possibly does not affect the arbitrariness condition. It is also notable that due to the nonlinearity including the material and geometrical nonlinearity, the arbitrariness is able to appear, it indicates that the fact that the governing equations for the deformation of composite materials are nonlinear equations is essential for the appearance of arbitrariness. Considering the actual deformation, the resultant displacement in the arbitrariness seems to have the following formula

$$
\dot{u}_{c}^{\prime}=v_{c}^{\prime} H_{L}\left(x_{1}^{\prime}-c\right)
$$

where the function $H_{L}\left(x_{1}^{\prime}\right)$ is the Heaviside function. Since theoretically arbitrary displacement is allowed, the width of the band of microbuckling is able to relate with the initial misalignment shape in the material around the 
area of initiation of the microbucling. When we put the tensor $A_{i j k l} n_{j} n_{l}$ as $a_{i k}$, the determinant of (30) is explicitly represented in two-dimensional as the following,

$$
\operatorname{det} a_{i k}=a_{11} a_{22}-a_{12} a_{21}=0
$$

In fiber reinforced composite materials, commonly the elastic modulus in fiber axial direction has much higher value than the value of transverse direction and stress value, and because of this, $C_{1111}^{\text {mat }}$ has much higher value than the other components of constitutive tensor $C_{i j k l}^{\text {mat }}$ and the components of stress tensor $\sigma_{i j}$, that is $C_{1111}^{\text {mat }} \gg C_{i j k l}^{\text {mat }}, \sigma_{i j}\left(C_{i j k l}^{\text {mat }} \neq C_{1111}^{\text {mat }}\right)$. Since only $A_{1111}$ and $a_{11}$ includes $C_{1111}^{\text {mat }}, A_{1111} \gg A_{i j k l} \quad\left(A_{i j k l} \neq A_{1111}\right)$ and $a_{11} \gg a_{i k}\left(a_{i k} \neq a_{11}\right)$. Thus the equation becomes,

$$
a_{22}=\frac{a_{12} a_{21}}{a_{11}} \approx 0
$$

Here the vector $n_{j}$ is represented using an angle $\beta$ as follows,

$$
n_{j}=\frac{\partial x_{1}^{\prime}}{\partial x_{j}}=\left(\begin{array}{ll}
\cos \beta & \sin \beta
\end{array}\right)
$$

Then $a_{22}$ is represented as follows,

$$
\begin{aligned}
a_{22}= & A_{2 j 2 l} n_{j} n_{l} \\
= & C_{2 j 2 l}^{\text {mat }} n_{j} n_{l}+\sigma_{j l} n_{j} n_{l} \\
= & \left(C_{2121}^{\text {mat }}+\sigma_{11}\right) \cos ^{2} \beta+\left(C_{2122}^{\text {mat }}+C_{2221}^{\text {mat }}+2 \sigma_{12}\right) \\
& \quad \cdot \cos \beta \sin \beta+\left(C_{2222}^{\text {mat }}+\sigma_{22}\right) \sin ^{2} \beta \approx 0
\end{aligned}
$$

From this equation,

$$
\begin{aligned}
-\sigma_{11} \approx C_{2121}^{m a t} & +\left(C_{2222}^{m a t}+\sigma_{22}\right) \tan ^{2} \beta \\
& +\left(C_{2122}^{m a t}+C_{2221}^{m a t}+2 \sigma_{12}\right) \tan \beta
\end{aligned}
$$

$-\sigma_{11}$ is the value of applied compressive stress to the material in longitudinal direction. When this applied stress reaches the value of right hand side of (38), the determinant of (30) becomes equal to zero, and the arbitrariness is allowed to appear, which means the instability appears in the material and microbuckling is able to occur in the actual situations. The value of $-\sigma_{11}$ at the time of being equal to right hand side of (38) is considered as the critical compressive stress $\sigma_{c r}$ or the buckling stress in microbuckling.

$$
\begin{aligned}
\sigma_{c r} \approx C_{2121}^{m a t} & +\left(C_{2222}^{m a t}+\sigma_{22}\right) \tan ^{2} \beta \\
& +\left(C_{2122}^{m a t}+C_{2221}^{m a t}+2 \sigma_{12}\right) \tan \beta
\end{aligned}
$$

Using elastic-plastic tangent shear modulus $G_{L T}^{e p}$, transverse tangent modulus $E_{T}^{e p}$, in-plane Poisson's ratio $v_{12}$ and $v_{21}$ and shear stress $\tau_{12}$, the equation becomes as follows,

$$
\begin{aligned}
\sigma_{c r} \approx G_{L T}^{e p}+ & \left(\frac{1}{1-v_{12} \nu_{21}} E_{T}^{e p}+\sigma_{22}\right) \tan ^{2} \beta \\
& +\left(C_{2122}^{\text {mat }}+C_{2221}^{\text {mat }}+2 \tau_{12}\right) \tan \beta
\end{aligned}
$$

In the case of uniaxial compression and if $C_{2122}^{\text {mat }}$ and $C_{2221}^{\text {mat }}$ are close to zero, the compressive strength is approximately represented as follows,

$$
\sigma_{c r} \approx G_{L T}^{e p}+\frac{1}{1-v_{12} v_{21}} E_{T}^{e p} \tan ^{2} \beta
$$

Equation (41) corresponds with the expression for longitudinal compressive strength of composite materials given by Budiansky [9]. It is indicated that the arbitrariness condition in equations of deformation of composite materials is closely related with the initiation condition of compressive failure of composite materials.

Moreover, the critical stress value is also represented by the properties of fibers and matrix. From (2),

$$
\begin{gathered}
G_{L T}^{e p} \approx G_{m}^{e p} \cdot \frac{\left(1-V_{f}\right)\left\{\left(G_{f L T}^{e}-G_{m}^{e p}\right) S_{L T}+G_{m}^{e p}\right\}+V_{f} G_{f L T}^{e}}{\left(1-V_{f}\right)\left(G_{f L T}^{e}-G_{m}^{e p}\right) S_{L T}+G_{m}^{e p}} \\
\approx G_{m}^{e p} \cdot \frac{\left\{\left(1-V_{f}\right) S_{L T}+V_{f}\right\} G_{f L T}^{e}+\left(1-V_{f}\right)\left(1-S_{L T}\right) G_{m}^{e p}}{\left(1-V_{f}\right) S_{L T} G_{f L T}^{e}+\left\{1-\left(1-V_{f}\right) S_{L T}\right\} G_{m}^{e p}}
\end{gathered}
$$

where $G_{m}^{e p}$ is the elastic-plastic tangent shear modulus of matrix, $G_{f L T}^{e}$ is the elastic in-plane shear modulus of fiber and $S_{L T}$ is the shear component of Eshelby tensor. When the shear modulus of fiber is much higher than the shear modulus of matrix $G_{f L T}^{e} \gg G_{m}^{e p}$,

$$
\begin{aligned}
G_{L T}^{e p} & \approx G_{m}^{e p} \cdot \frac{\left\{\left(1-V_{f}\right) S_{L T}+V_{f}\right\} G_{f L T}^{e}}{\left(1-V_{f}\right) S_{L T} G_{f L T}^{e}} \\
& \approx G_{m}^{e p} \cdot\left(1+\frac{V_{f}}{1-V_{f}} S_{L T}^{-1}\right)
\end{aligned}
$$

The Eshelby tensor depends on the geometrical shape of reinforcement fibers. Here, two kinds of fibers shown in Fig. 5 are assumed. The case 1 in Fig. 5 is the case where fibers and matrix have plate shape, and the case 2 in Fig. 5 is the case where fibers are cylinder solids and matrix surrounds fibers. In case 1, the value of Eshelby tensor in

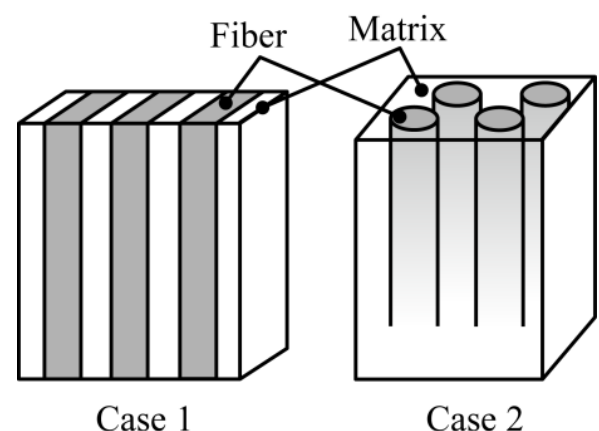

Figure 5. Two kinds of model of composites 
shear component is $S_{L T}=1$. Then

$$
\begin{aligned}
& G_{L T}^{e p} \approx G_{m}^{e p} \cdot\left(1+\frac{V_{f}}{1-V_{f}}\right)=\frac{G_{m}^{e p}}{1-V_{f}} \\
& \sigma_{c r} \approx \frac{G_{m}^{e p}}{1-V_{f}}
\end{aligned}
$$

On the other hand, in case $2, S_{L T}=1 / 2$. Then

$$
\begin{gathered}
G_{L T}^{e p} \approx G_{m}^{e p} \cdot\left(1+\frac{2 V_{f}}{1-V_{f}}\right)=\frac{1+V_{f}}{1-V_{f}} G_{m}^{e p} \\
\sigma_{c r} \approx \frac{1+V_{f}}{1-V_{f}} G_{m}^{e p}
\end{gathered}
$$

In addition, the elastic-plastic tangent shear modulus of matrix is closely related with the current yield state of matrix. From (3),

$$
d \tau_{m} \approx \frac{S_{L T} G_{f L T}^{e}+\left(1-S_{L T}\right) G_{m}^{e p}}{\left\{V_{f}+\left(1-V_{f}\right) S_{L T}\right\} G_{f L T}^{e}+\left(1-V_{f}\right)\left(1-S_{L T}\right) G_{m}^{e p}} d \tau_{12, \text { comp }}
$$

where $d \tau_{m}$ is shear stress rate of matrix and $d \tau_{12, \text { comp }}$ is applied shear stress rate of composites. When the shear modulus of fiber is much higher than the shear modulus of matrix $G_{f L T}^{e} \gg G_{m}^{e p}$,

$$
\begin{gathered}
d \tau_{m} \approx \frac{S_{L T} G_{f L T}^{e}}{\left\{V_{f}+\left(1-V_{f}\right) S_{L T}\right\} G_{f L T}^{e}} d \tau_{12, \text { comp }} \\
\approx \frac{1}{1-V_{f}+V_{f} S_{L T}{ }^{-1}} d \tau_{12, \text { comp }} \\
d \tau_{12, \text { comp }} \approx\left(1-V_{f}+V_{f} S_{L T}^{-1}\right) d \tau_{m}
\end{gathered}
$$

Then considering the integration until the time when the significant degradation of tangent modulus occurs,

$$
\tau_{12} \approx\left(1-V_{f}+V_{f} S_{L T}^{-1}\right) \tau_{m Y}
$$

where $\tau_{12}$ is applied shear stress to composites and $\tau_{m Y}$ is yield stress of matrix. Generally the fibers have a slight misalignment, and this misalignment affects the local stress distribution of the material. Considering the equilibrium condition of applied stress in between misalignment coordinate system and coordinate system associated with global fiber direction,

$$
\begin{gathered}
\sigma_{x x} \phi+\tau_{x y}=\tau_{12} \\
\sigma_{c r}=\frac{\tau_{12}-\tau_{x y}}{\phi} \approx \frac{\left(1-V_{f}+V_{f} S_{L T}^{-1}\right) \tau_{m Y}-\tau_{x y}}{\phi}
\end{gathered}
$$

where $\sigma_{x x}$ and $\tau_{x y}$ are stress in longitudinal direction and shear stress in the coordinate system associated with global fiber direction, respectively and $\phi$ is the misalignment angle of fiber. From this equation, the relationship of compressive strength with matrix yield stress, applied shear stress, and fiber volume fraction are represented as the linear relation, and the relationship with misalignment of fiber is represented as inversely proportional. In addition, the dependency of compressive strength for the fiber volume fraction $V_{f}$ is related with the shear component of Eshelby tensor $S_{L T}$. When fibers are plates, $S_{L T}=1$ and

$$
\sigma_{c r}=\frac{\tau_{m Y}-\tau_{x y}}{\phi}
$$

When fibers are cylinder solids, $S_{L T}=1 / 2$ and

$$
\sigma_{c r}=\frac{\left(1+V_{f}\right) \tau_{m Y}-\tau_{x y}}{\phi}
$$

\section{Numerical Analysis for Longitudinal Compressive Strength Using Arbitrariness Condition}

Here the numerical analysis is conducted for the actual material property using the arbitrariness condition. For this purpose, incremental analysis is conducted. As the initial condition, stress is set to zero. Then the stress is incrementally applied. In each increment, total stress is calculated and matrix plastic state is updated. Constitutive tensors of fiber, matrix, and composites are calculated, and the determinant in (30) is evaluated. When the determinant in (30) becomes approximately equal to zero, the arbitrariness is assumed to occur, and the microbuckling is assumed to initiate. At this increment, the calculation is finished, and the applied compressive stress at this time is recorded as the material strength or the microbuckling stress. For transverse failure modes, failure criteria presented by Pinho et al. [10] are applied. The material property of carbon fiber reinforced plastics (CFRP) AS4/3501-6 [6] is assumed. For strain hardening curve of matrix, two kinds of hardening curves $\mathrm{M}$ and $\mathrm{N}$ shown in Fig. 2 are applied and the results are compared. The analysis is repeated with changing each one parameter, and the results for the relationship between material strength and each one parameter are obtained.

Fig. 6(a) shows the analysis results for relationship between compressive strength and the multi-axial stresses. The shear stress reduces the compressive strength and this relation is approximately represented as the linear relation. Tensile and high compressive transverse stress also reduces the compressive strength, while under the small compressive transverse stress, the compressive strength is almost constant. In addition, the dependency of the multiaxial stresses changes with the change of the strain hardening curve. Fig. 6(b) shows the analysis results for the relationship between compressive strength and the constituent material property. The matrix yield stress and fiber volume fraction increases the compressive strength and these relations are also close to the linear relation. The initial fiber misalignment reduces the compressive strength and this relation is close to the inversely proportional relation. The dependency of the material strength for each parameter almost agrees with the experimental results shown in the previous investigations [3-4]. 


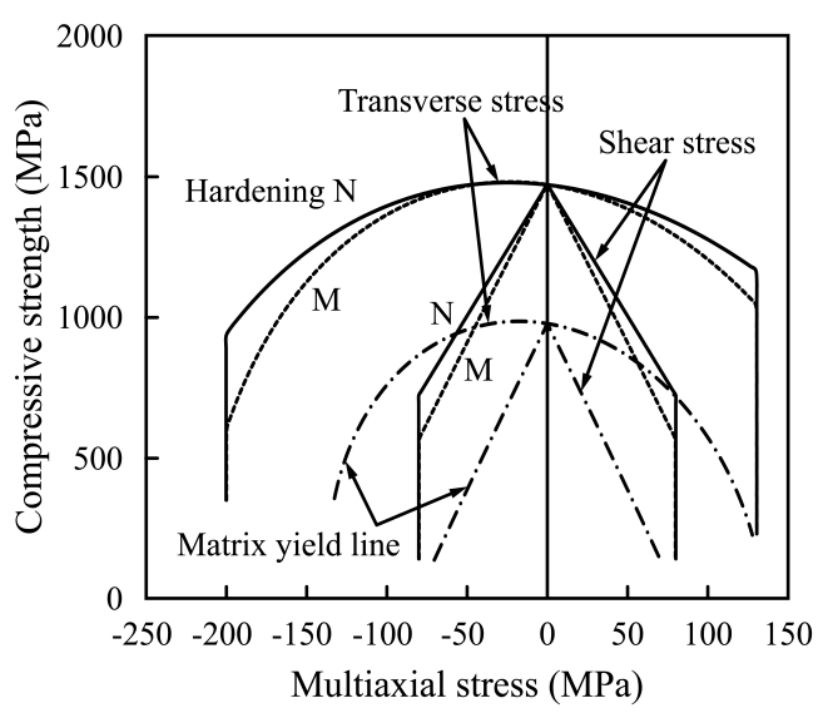

(a) Effect of multi-axial stress

Initial misalignment (deg)

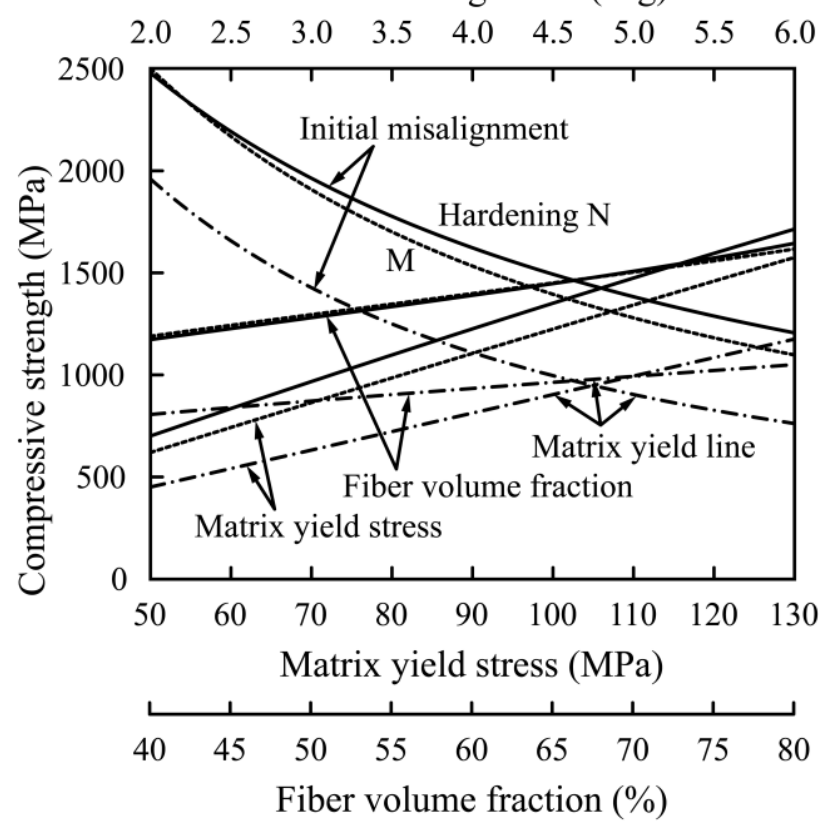

(b) Effect of material property

Figure 6. Numerical results for compressive strength in longitudinal compressive failure.

\section{NUMERICAL SIMULATION OF COMPRESSIVE FAILURE IN QUASI-ISOTROPIC LAMINATE}

\section{A. Numerical Model}

Finally the numerical simulation of compressive failure in quasi-isotropic laminate is conducted. Fig. 7 shows the numerical model of the compressive failure in quasiisotropic laminate. The stacking sequence of the laminate is [45/0/-45/90]s symmetric laminate. The thickness of each ply is $145 \mu \mathrm{m}$. The length in $\mathrm{X}$-direction of the model is $500 \mu \mathrm{m}$, and the thickness in z-direction is $100 \mathrm{~mm}$. The diameter of each fiber is set to $7.0 \mu \mathrm{m}$, and the interval of fibers in 0 -degree plies are $10.0 \mu \mathrm{m}$. The fiber volume fra-

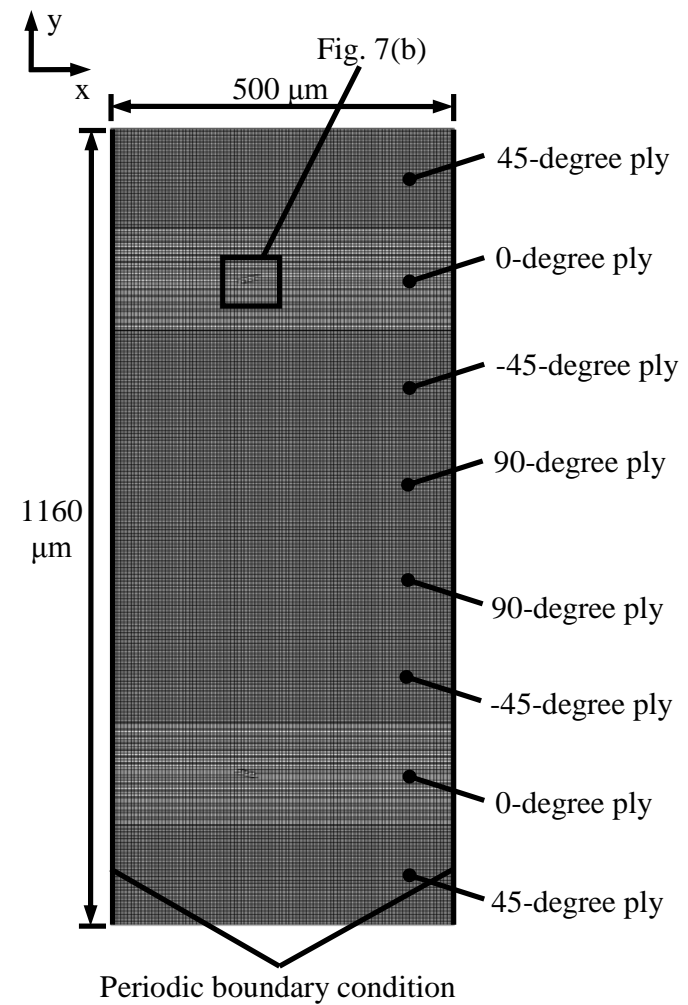

(a) Numerical model of quasi-isotropic laminate

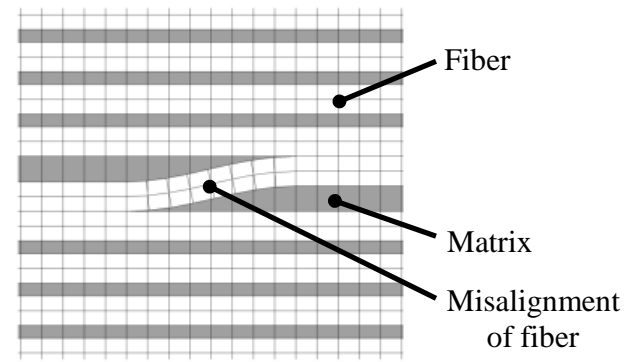

(b) Initial misalignment of fiber

Figure 7. Numerical model for compressive failure of quasi-isotropic laminate.

TABLE III. MATERIAL PROPERTY OF \pm 45 -DEGREE PliES [6]. CFRP AS4/3501-6 IS ASSUMED [6].

\begin{tabular}{|l|c|c|}
\hline Elastic modulus in x-direction & 61 & $\mathrm{GPa}$ \\
\hline Elastic modulus in y-direction & 11 & $\mathrm{GPa}$ \\
\hline In-plane Poisson's ratio & 0.28 & \\
\hline In-plane shear modulus & 5.8 & $\mathrm{GPa}$ \\
\hline
\end{tabular}

TABLE IV. MATERIAL PRoperty OF 90-DEgREe Plies [6]. CFRP AS4/3501-6 IS ASSUMED [6].

\begin{tabular}{|l|c|c|}
\hline Elastic modulus in $\mathrm{x}$ and y-direction & 11 & $\mathrm{GPa}$ \\
\hline In-plane Poisson's ratio & 0.40 & \\
\hline In-plane shear modulus & 4.3 & $\mathrm{GPa}$ \\
\hline
\end{tabular}

ction of the materials is set to $60.0 \%$, and there are 15 fibers in each 0-degree ply. Similar to the analysis in Sec. II, the initial misalignment of fiber is introduced as shown 


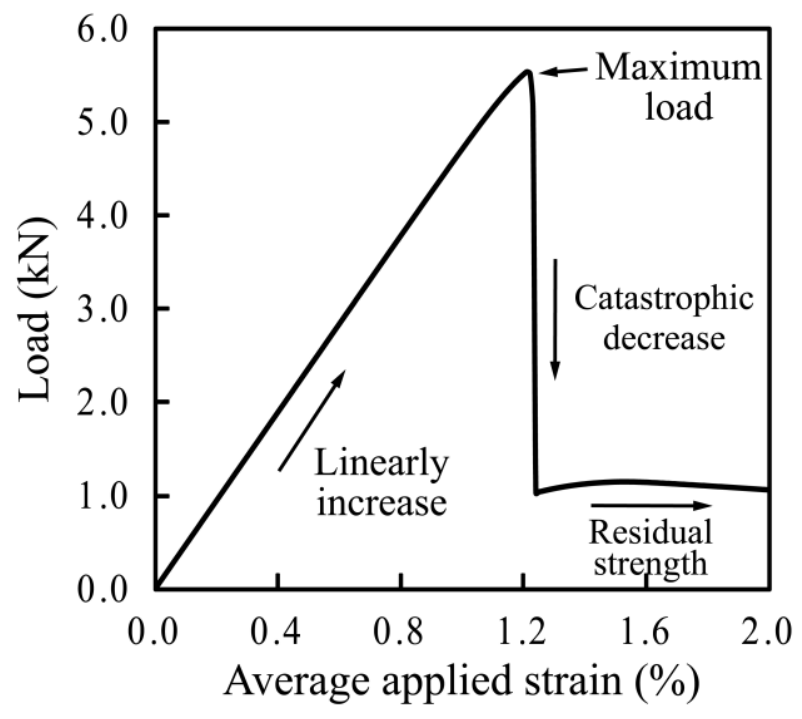

Figure 8. Simulated result of load-strain curve.

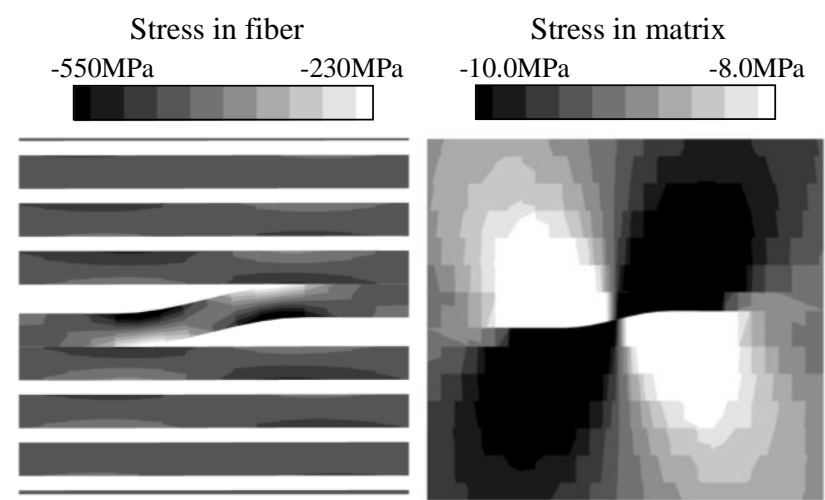

Average applied strain $0.20 \%$

Figure 9. Simulated results of stress distribution in 0-degree ply.

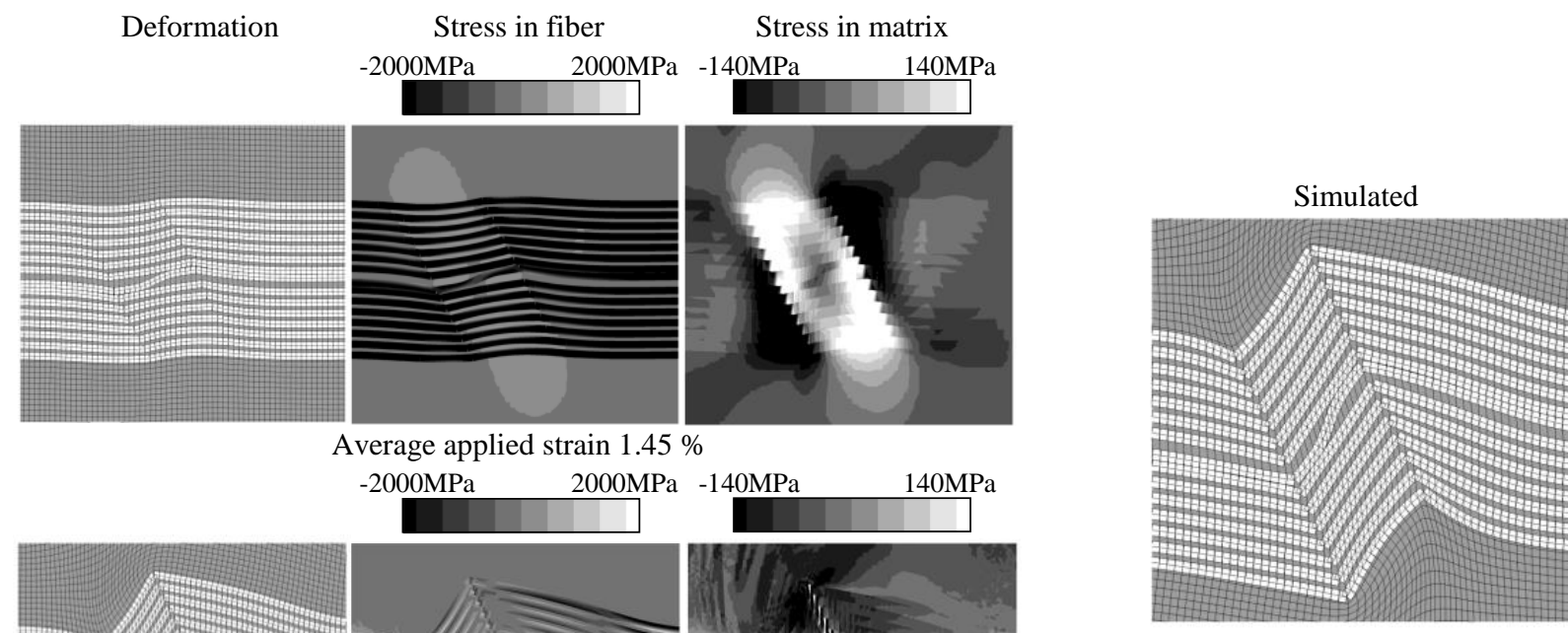

Experimental (Microscope picture)

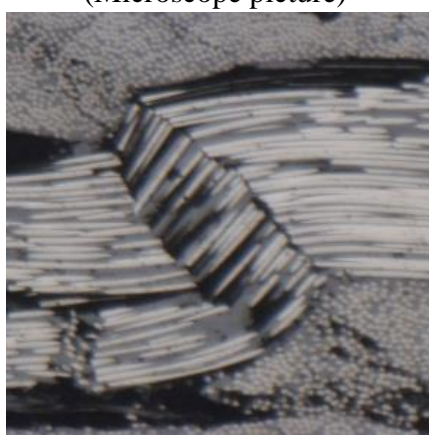

(b) Comparison with experimental result

in Fig. 7(b). The periodic boundary condition is introduced in both edge of the laminate to avoid the edge fracture of the material. The average applied strain in the initial increment is set to $0.004 \%$. In this analysis, in order to 
simulate the bending breaking of each fiber, the fibers in 0degree plies are modeled by circle cross-section beam elements, and the cohesive elements are inserted in the connection of fiber beam elements. When the high bending deformation occurs in fiber beam elements, the cohesive elements open and the fiber bending breaking occurs in the analysis. The fiber beam elements applied in this analysis are the Timoshenko type beam elements and have three nodes and four integration points. The matrix in 0-degree plies and \pm 45 , 90-degree plies are modeled by twodimensional plate elements. $\pm 45,90$-degree plies are modeled as the homogeneous composite materials, and the individual fibers and matrix are not modeled here. The material property for \pm 45 and 90 -degree plies used in this analysis is shown in Table III and IV [6].

\section{B. Simulated Results and Discussions}

Fig. 8 shows the simulated result of load-strain curve. After starting the loading, the load response is initially close to linear. When the load value increases, there is a slight nonlinearity in load-strain curve. The reason of the slight nonlinearity is considered to be due to the slight rotation of fibers in 0 -degree plies during the loading because of the misalignment of fibers. Due to the misalignment, when the load is applied the fibers in 0degree plies slightly rotate, then the average elastic modulus of the laminate in $\mathrm{x}$-direction slightly reduces during the loading, consequently the nonlinearity causes in load-strain curve. When the load value reaches $5.6 \mathrm{kN}$, and the average applied strain is $1.20 \%$, the load is catastrophically decreasing in load-strain curve, and after this decrease the load level keeps low level. The maximum supported load value in load-strain curve is recorded to be $5.6 \mathrm{kN}$.

Fig. 9 shows the stress distribution in 0-degree ply at strain $0.20 \%$. As shown in Fig. 9, the stress concentration occurs around the initial misalignment of fiber in 0-degree plies. At strain $1.20 \%$, the fiber bending breaking initiates in 0-degree plies. After the initiation of fiber bending breaking in 0-degree plies, the damage gradually develops as the applied strain increases, and the final fracture is formed as shown in Fig. 10. After the significant load decrease in load-strain curve, the load value keeps low level during the damage evolution, which is considered as the residual strength of the material. Fig. 10(b) shows the comparison between the simulated failure and the microscope picture observed in the experiment. The deformation after the failure of the material in the analysis agrees with the microscope picture of the experimental result.

\section{CONCLUSIONS}

The numerical simulation model and theoretical model for longitudinal compressive failure are obtained. In the numerical simulation, the deformation after the failure of the material agrees with the microscope picture of the experimental results. In theoretical modeling, the critical stress value is represented in several forms. The present analysis models are applicable for computer analysis of material strength.

\section{REFERENCES}

[1] J. LLorca, C. González, J. M. Molina-Aldareguía, J. Segurado, R. Seltzer, F. Sket, M. Rodríguez, S. Sádaba, R. Muñoz, and L. P. Canal, "Multiscale Modeling of Composite Materials: a Roadmap Towards Virtual Testing," Adv. Mater., vol. 23, pp. 5130-5147, 2011.

[2] M. J. Hinton and P. D. Soden, "Predicting Failure in Composite Laminates: The Background to The Exercise," Compos. Sci. Technol., vol. 58, pp. 1001-1010, 1998.

[3] C. R. Schultheisz and A. M. Waas, "Compressive Failure of Composites, Part I: Testing and Micromechanical Theories,” Prog. Aerosp. Sci., vol. 32, pp. 1-42, 1996.

[4] A. M. Waas and C. R. Schultheisz, "Compressive Failure of Composites, Part II:, Experimental Studies” Prog. Aerosp. Sci., vol. 32, pp. 43-78, 1996.

[5] S. Basu, A. M. Waas, and D. R. Ambur, "Compressive Failure of Fiber Composites Under Multi-axial Loading," J. Mech. Phys. Solids, vol. 54, pp. 611-634, 2006

[6] P. D. Soden, M. J. Hinton, and A. S. Kaddour, "Lamina Properties, Lay-up Configurations and Loading Conditions for a Range of Fibre-Reinforced Composite Laminates," Compos. Sci. Technol., vol. 58, pp. 1011-1022, 1998.

[7] C. S. Yerramalli and A. M. Waas, "A Failure Criterion for Fiber Reinforced Polymer Composites Under Combined CompressionTorsion Loading," Int. J. Solids Struct., vol. 40, pp. 1139-1164, 2003.

[8] K. Tohgo, Y. Sugiyama, and K. Kawahara, "Ply-Cracking Damage and Nonlinear Deformation of CFRP Cross-Ply Laminate," JSME Int. J., Series A, vol. 45, pp. 545-552, 2002.

[9] B. Budiansky, "Micromechanics," Comput. Struct., vol. 41, pp. 312, 1983.

[10] S. T. Pinho, C. G. Davila, P. P. Camanho, L. Iannucci and P. Robinson, "Failure Models and Criteria for FRP under In-plane or Three-dimensional Stress States Including Shear Non-linearity," NASA Technical Memorandum-2005-213530, 2005. 